\title{
EFFECTS OF POLICOSANOL ON CELLS INVOLVED IN ATHEROSCLEROSIS DEVELOPMENT.
}

\author{
Noa M*, Más R*., de la Rosa M.C.**, Falcón V.** \\ *Pharmacology Dpt, Center for Natural Products, CNIC, P.O.Box 6414, Havana, Cuba. \\ **Electron Microscopy Lab, CIGB, Havana, Cuba.
}

Atherosclerosis is the principal cause of cardiovascular, cerebrovascular and peripheral artery disease. Rupture of the plaque surface and subsequent luminal thrombus formation is probably the most important mechanism underlying the acute coronary syndromes A very important factor that makes a plaque vulnerable to rupture is the presence of activated macrophages and the formation of foam cells indicating ongoing inflammation. These cells are capable of digesting extracellular matrix by direct phagocytosis or by secreting several proteolytic enzymes such as plasminogen activators, tissue necrosis factor alpha and a variety of matrix metalloproteinases, resulting in thinning and further weakening of the plaque, predisposing it to the rupture.

Neointimal formation due to smooth muscle cell (SMC) proliferation is a crucial step in the progression of the atherosclerotic plaque. Accelerated proliferation of smooth muscle cell also appears to be a cause of early coronary occlusion on patients undergoing heart transplantation, coronary artery bypass graft and percutaneous transluminal coronary angioplasty. Thus, factors controlling SMC proliferation are important for atherosclerosis development.

Most of inhibitors of hydroxy methylglutaryl CoA reductase (statins), a class of cholesterollowering drugs inhibiting cholesterol biosynthesis, reduce the number of inflammatory cells and prevent SMC proliferation both in vivo and in vitro, this effect being considered one of the pleiotropic effects of these drugs.

Policosanol is a mixture of higher aliphatic primary alcohols purified from sugar cane (Saccharum officinarum, L) wax, lowering cholesterol by inhibiting cholesterol biosynthesis at a step located between acetate consumption and mevalonate formation. This inhibition is achieved through an indirect regulation, instead of a competitive inhibition, of the enzymatic activity of HMGCoA reductase. The cholesterol-lowering effects of policosanol have been demonstrated in patients with type II hypercholesterolemia and in the dyslipidemia due to type 2 diabetes.

Previous works using Electron Microscopy (EM) have shown that policosanol reduces lipofundininduced atherosclerotic lesions in rabbits and rats, including foam cell formation [1], and prevents the development of foam cells in carrageenan-induced granulomas in rats [2] (Figs 1, 2). Likewise, policosanol prevented the spontaneous development of atherosclerotic lesions and reduce the number of macrophages identified by immunohistochemistry in Macaca arctoides monkeys . The effects of policosanol on the development of atherosclerotic lesions have been also shown in rabbits fed a cholesterol-rich diet, despite in this model it was not able to significantly prevent the increase on serum cholesterol elicited by the diet.

Policosanol also inhibited SMC proliferation induced in rabbit cuffed artery, as revealed by inmunohistochemical detection of proliferative cell nuclear antigen [3,4]. The different phenotypes 
of SMC and the apoptosis of these cells were studied by EM (Fig 3). Furthermore, indirect experimental and clinical evidence has indicated that policosanol exerts protective effects on endothelium. [5].

Taking into account that policosanol exerts a direct antiatherosclerotic effect in the arterial wall beyond their lipid lowering effect, such pleiotropic action could be of great value in the prevention of cardiovascular disease.

\section{References}

[1] Noa M. et al., J Pharm Pharmacol 47 (1995): 289.

[2] Noa M. et al., J Pharm Pharmacol 48 (1996): 306.

[3] Noa M. et al , Int. J. of Cardiology.67 (1998):125

[4] Noa M. et al., J Pharm Pharmacol 49 1997: 999.

[5] Castaño G. et al., Int.J.Clin.Pharmacol.Res 4 1999:105.

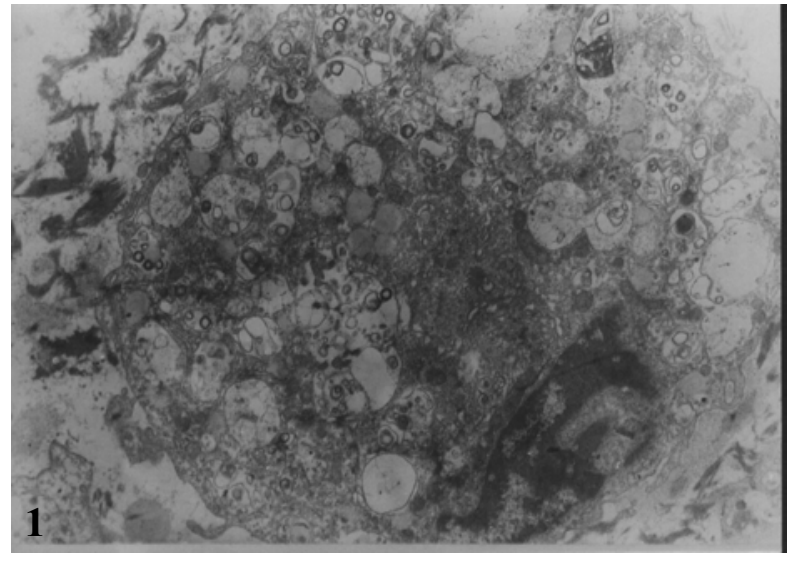

A control group developing foam cell with large lysosomal carrageenan inclusions, more than 10 lipid inclusions and some myelin forms (10 200 X)

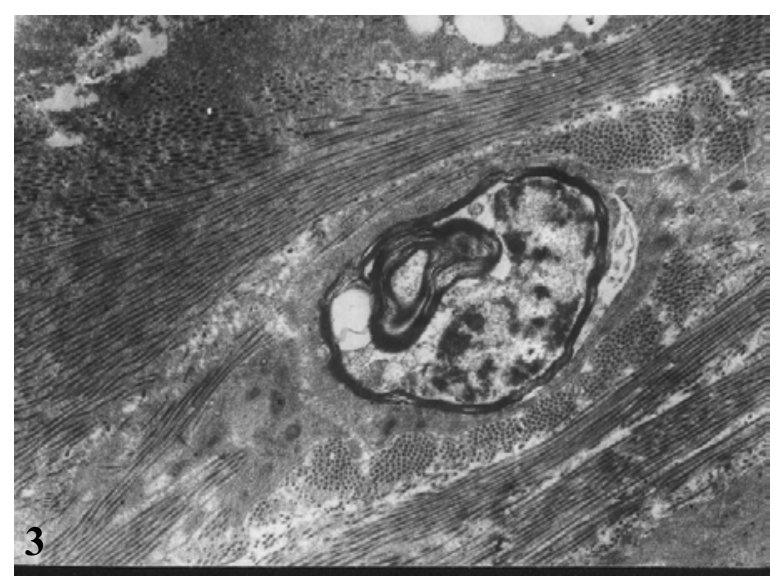

An apoptotic cell in the neointima of a cuffed carotid artery from a control rabbit $(8000 \mathrm{X})$

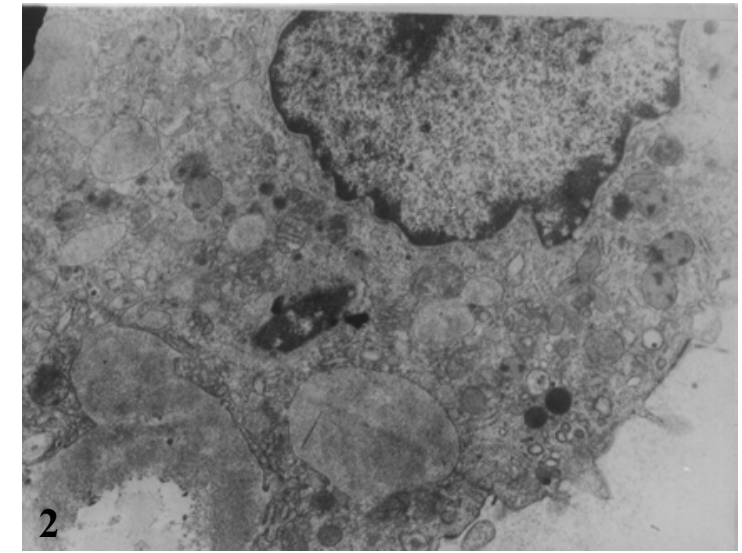

A policosanol treated macrophage showing some lysosomal carrageenan inclusions. No lipids inclusions are seen $(16000 \mathrm{X})$ 